le portiQue $\begin{array}{ll}\text { Le Portique } \\ \text { Revue de philosophie et de sciences humaines }\end{array}$

$10 \mid 2002$

Les paradis artificiels

\title{
La débrouille des familles
}

\section{Pascale Jamoulle}

\section{(2) OpenEdition}

\section{Journals}

Édition électronique

URL : http://journals.openedition.org/leportique/138

DOI : 10.4000/leportique.138

ISSN : $1777-5280$

\section{Éditeur}

Association "Les Amis du Portique"

Édition imprimée

Date de publication : 1 septembre 2002

ISSN : 1283-8594

\section{Référence électronique}

Pascale Jamoulle, "La débrouille des familles », Le Portique [En ligne], 10 | 2002, mis en ligne le 06 juin 2005, consulté le 09 avril 2021. URL : http://journals.openedition.org/leportique/138 ; DOI : https:// doi.org/10.4000/leportique.138

Ce document a été généré automatiquement le 9 avril 2021.

Tous droits réservés 


\title{
La débrouille des familles
}

\author{
Pascale Jamoulle
}

1 Une étude ethnographique auprès de familles belges de milieu populaire, traversées par des conduites à risques (violences, micro-trafics, tentatives de suicide, addictions...), a fait émerger leur parole et leur regard sur leur histoire ${ }^{1}$. Parents, fratries, proches racontent la vie ordinaire dans des quartiers aux marges de la mondialisation, où l'économie souterraine capte les jeunes «entrepreneurs» et structure une part toujours plus importante des rapports sociaux. Les tensions et les mises en danger de la jeunesse y sont souvent des conduites d'adaptation au fonctionnement des lieux de socialisation (vie domestique, sociabilités de quartier, milieu scolaire). Les récits se croisent sur des désordres familiaux, socio-économiques, scolaires et judiciaires. Dans ces contextes, des familles se débrouillent, sortent de l'isolement, trouvent des ressources dans leur environnement et font preuve d'ingéniosité pour faire face à leur condition. Elles explorent les appuis qu'elles ont trouvés dans le réseau social et les dispositifs d'aide pour faire évoluer leurs contextes de vie et leurs «intrigues familiales ».

2 Quel est le sens des prises de risques démesurées qui traversent les familles? Addictions, violences, micro-trafics, auto-mutilations, tentatives de suicide... sont autant de questions posées aux adultes et à la société. "Celui qui n'est pas passé par là ne peut pas comprendre " disent souvent les familles, "ce sont des problèmes qui touchent le sens de la vie. On est atteint, le sens de la vie est atteint. »Cette enquête de terrain explore leurs vécus intimes et collectifs.

Une approche ethnographique

3 Pendant deux années j'ai mené une enquête de terrain auprès de dix-huit familles de milieu populaire débordées par les conduites à risques d'un des leurs. Je l'ai localisée dans deux anciens sites miniers du Hainaut belge, où les déterminants sociaux des mises en danger et des tensions que vit la jeunesse ont une lisibilité particulière. Pendant l'époque industrielle, cette région fut un des fleurons des luttes ouvrières. La restructuration de la sidérurgie et des constructions métalliques a commencé dans les années 1970 alors que les derniers charbonnages fermaient. Elle a fragilisé la population non qualifiée (baisse drastique de la probabilité d'emploi, désagrégation des 
réseaux sociaux autour du travail). Parallèlement, les pratiques économiques souterraines s'implantaient dans le tissu social. Actuellement les anciens corons et cités ouvrières rassemblent, "aux marges » de l'économie mondialisée, les personnes en situation de fragilité familiale, économique, sociale et judiciaire.

4 Par un lent processus d'imprégnation et grâce à des sources variées (observations, conversations informelles, entretiens), j'ai cherché à recomposer les réalités quotidiennes et les parcours de familles rencontrées ${ }^{2}$. Toxicomanies et micro-trafics traversent la plupart des récits. Je les ai analysés à travers le prisme plus large des "conduites à risques", les trajectoires familiales de mes interlocuteurs faisant largement apparaître, simultanément ou en déplacement successif, un ensemble de comportements "décalées ", destructeurs ou auto-destructeurs, qui fragilisent les individus, traversent les univers domestiques et procèdent des mêmes substrats et styles de vie.

5 En réalisant des récits de vie, à but de recherche, les familles sont devenues auteurs dans le sens où elles se sont autorisées à dire ce qui ne peut l'être ordinairement ${ }^{3}$. Elles ont développé leurs facultés d'expression et de traduction de leurs itinéraires, prenant confiance dans l'échange social et leur propre capacité de s'y impliquer.

L'espace social

6 À force d'interroger les métaphores de mes interlocuteurs, d'aligner les scénarios familiaux et de les croiser, j'ai fini par entrevoir le filigrane de l'histoire sociale. Les prises de risques démesurées qui traversent ces familles étaient des conduites d'adaptation aux principaux lieux de socialisation des jeunes : le quartier, la famille et l'école.

Les sociabilités de quartier

7 Mes sites d'enquête sont principalement constitués d'une mosaïque de clos ou blocs de logements sociaux, construits à l'époque du plein emploi, pour stabiliser les populations ouvrières. Actuellement ils sont surtout habités par des personnes retraitées ou des familles vivant d'allocations sociales. Les loyers sont proportionnels au revenu. Les habitants qui ont un travail déclaré quittent ces cités. Prioritaires dans l'octroi des logements, les familles monoparentales sont sur-représentées. Beaucoup ne disposent pas de véhicule. Il n'y a ni commerce ni services publics dans ces sites, exclusivement locatifs et isolés du centre urbain. Il existe, par contre, une économie de proximité, clandestine, très diversifiée. Les «business" (pratiques économiques illégales) font partie de la vie quotidienne. Tout se vend et s'achète «moins cher » sur les pas-de-porte ou dans les lieux publics. Les populations «se débrouillent » pour faire face «aux problèmes d'argent » qui se posent en permanence. Dans le contexte de prohibition des stupéfiants, les activités liées aux drogues sont un terrain d'aventures entrepreneurial ${ }^{4}$. Elles s'inscrivent dans les sociabilités de quartier et permettent aux jeunes de s'associer, "de se faire valoir ", de protéger leur famille, de "faire l'Américain " et de se bricoler une forme de participation au modèle culturel postmoderne ${ }^{5}$.

8 Si une part du business est gérée par les réseaux familiaux et s'appuie sur les liens de parenté, la plupart des familles assistent, impuissantes, à l'expérimentation puis à l'insertion des adolescents dans les échanges socio-économiques illégaux. Ils deviennent le lieu de socialisation ${ }^{6}$ principal d'une part de la jeunesse. Les adolescents $\mathrm{y}$ intègrent le système de normes, de valeurs et de conduites (sociales, économiques et symboliques) de la culture de la rue ${ }^{7}$. 
9 Le savoir des parents n'a plus beaucoup de crédit aux yeux d'une partie de ces jeunes «entrepreneurs". Pour les familles issues de l'immigration, le choc est particulièrement rude. Au pays, les «anciens » transmettaient la coutume, ils étaient respectés. Dans les rapports sociaux de l'économie souterraine, jeunes et vieux sont soumis à la loi du plus fort. Les positions y sont incertaines et les arbitres manquants. La domination s'exerce dans le «business» comme dans la vie privée. La violence sexuelle est parfois banalisée, surtout envers les jeunes filles vulnérables, dont les liens de parenté et d'interconnaissance sont peu étendus.

10 Certaines pratiques culturelles comme la "tchache ${ }^{8}$ ou le hip hop permettent de représenter les frustrations, les désirs et autres jeux d'affects, de construire du symbolique et de diminuer la violence intestine. En revanche, l'esthétique du pulsionnel, du « hard grave ${ }^{9}$, qui se diffuse dans les quartiers, institue les passages à l'acte violents comme les seuls modes d'expression possibles.

Dans mes sites d'enquête, la lutte des classes est devenue la lutte des " castes ", avec d'autres mécanismes de clivages sociaux ${ }^{10}$. Les distances se creusent entre les personnes qui ne partagent pas la même condition : entre les habitants du quartier et les extérieurs, entre les représentants institutionnels, "les deuxièmes classes du Titanic ", et les usagers des services, «les troisièmes classes ». Les populations se classent en «forts en mentalité » (les battants) et « faibles en caractère » (les perdants), « caïds » et " toxines » (toxicomanes)... Entre les « castes », il y a peu d'empathie, de nuances, de ponts.

12 Pris dans une appropriation locale du modèle américain, des jeunes s'essayent sur les drogues, les plans de "business » et autres pratiques à risques pour se construire une réputation dans le quartier et se mettre à l'épreuve, apprendre par la pratique s'ils sont du côté des forts ou du côté des faibles. Dans des contextes de concurrence exacerbée, où la plupart en restent « aux petites commissions et maxi risques » et rêvent de conquérir des parts de marché, le climat est explosif, les populations vivent dans l'insécurité et la violence.

Les vies domestiques

13 Les trames des récits révèlent des histoires familiales apparentées. Dans les univers domestiques où j'ai été invitée, les familles s'adaptent dans le fracas aux transformations de la famille patriarcale. La plupart des pères ont disparu ou sont très disqualifiés. Certains ont été mis à l'écart à cause de leur violence ou de leur intolérance. D'autres habitent encore la maison familiale, mais y sont comme transparents, effacés par l'intensité des rancœurs de leur femme. Mes interlocutrices ${ }^{11}$ sont intarissables sur leurs insatisfactions conjugales, "le manque de discuter" et l'immaturité de ces pères qui ne subviennent pas aux besoins affectifs et économiques de leurs enfants et en n'ont pas d'autorité sur eux. Les pères sont le point aveugle de l'enquête, ceux dont on ne sait rien sinon la place immense qu'ils prennent, par défaut, en creux, dans les relations familiales. Les grands-mères et les mères ont trouvé du sens dans l'éducation et, parfois, l'appropriation des enfants. Où les pères ont-ils trouvé du sens? Qu'est-ce qui les a fait fuir? Autant de questions que l'enquête laisse sans réponse.

14 Dans les univers sans hommes où j'ai été invitée, les femmes doivent «être le père et la mère à la fois ", mais, souvent, elles n'y arrivent pas et l'un des enfants prend auprès d'elles une place affective qui ne lui revient pas. Au côté de mères parfois très envahissantes grandissent de "petits hommes de la maison» (ou des adolescentes) qui veulent soumettre leur famille à leur loi. Lorsque des liens trop serrés lient ces jeunes à 
leur mère, les relations sont violentes. Pris dans ce trop plein d'amour maternel, ils se sentent impuissants à s'émanciper et leurs colères sont sans limite. Le sentiment d'injustice et de révolte des fratries, qui se vivent comme les laissées pour compte de l'amour parental, crée des états de guerre familiaux et multiplie les conduites d'appel des jeunes.

Les huis-clos domestiques sont d'autant plus oppressants qu'il y a peu de régulations externes. Beaucoup d'interlocutrices sont en conflit, voire en rupture définitive avec leur famille élargie. Enfermées par leurs problèmes de santé et leurs conditions de vie précaires, elles ont peu de contact avec le voisinage et la communauté. On peut comprendre ce que représentent, pour ces jeunes, pris dans ces clôtures relationnelles et économiques, les sociabilités de la rue et les conduites liées aux drogues : un espace ludique où s'associer, une liberté, une émancipation, un accès à la société de consommation.

Le milieu scolaire

16 La précarité socio-économique et les désordres familiaux ont des effets sur les enfants. L'atteinte la plus courante est le refus d'apprendre et le mépris affiché pour les adultes du milieu scolaire ${ }^{12}$. Aux prises avec la logique de sélection scolaire, de nombreux enfants vivant dans mes quartiers d'enquête ont basculé de l'enseignement général vers le technique, le professionnel, l'alternance ou le spécial. Certaines écoles accueillent majoritairement des jeunes qui ont fait des apprentissages sociaux dans la culture de la rue. Les équipes éducatives sont de plus en plus confrontées à des adolescents qui veulent leur part de "business " de quartiers, être "les petits hommes " de la maison et les "petits caïds» à l'école. Ces écoles sont le théâtre de luttes quotidiennes entre les codes conduites de la culture dominante, qu'elles sont censées transmettre, et le système de normes contraignants de la culture de la rue. Par moment le monde scolaire se fragmente, prend peur, dissimule ses impossibilités socioéducatives et s'épuise. L'absentéisme des enseignants répond à celui des élèves. Des trous béants apparaissent dans l'obligation scolaire. La violence et les transgressions se multiplient dans un sentiment d'impuissance généralisé. « Le cercle de l'indifférence » des équipes et la scène de la rue peuvent prendre le pas sur l'enseignement. Dans d'autres situations, les équipes éducatives parviennent à garantir la paix scolaire, à protéger les élèves et les enseignants les plus vulnérables, et à offrir une réelle qualification à ces jeunes. Certaines déploient une énergie considérable, en interne et en externe, pour prévenir les parcours de marginalisation de la jeunesse. Elles vont à domicile, nouent des contacts avec les familles les plus exposées et cherchent à soutenir leurs compétences.

L'accès aux institutions

17 Notre structure sociale a professionnalisé la gestion des affects et des douleurs. L'État met à la disposition des familles des structures et des intervenants psycho-médicosociaux. Si les récits dévoilent beaucoup de troubles du "trop proche », les relations des familles avec les professionnels relèvent, elles, souvent, de troubles du «trop lointain ». Les langages verbaux et non verbaux, les vécus, les manières d'engager une relation, de se saluer, de se montrer affecté sont très différents. Pour une catégorie de familles, les intervenants appartiennent à une autre «bulle» sociale. Elles franchissent difficilement la porte des structures d'aide. Elles se sentent des étrangères dans les univers institutionnels. 

d'entrée. Où peuvent-elles s'adresser quand elles se sentent débordées par leurs relations familiales? Les services psychiatriques ou de santé mentale sont marqués par le champ sémantique de la folie ${ }^{13}$. Quant aux structures pour toxicomanes, y demander de l'aide, c'est déjà classer le jeune dans la «basse classe » et risquer de stigmatiser l'ensemble de la famille.

19 Parfois l'extérieur est vécu comme hostile et le système relationnel qui s'est instauré est cadenassé. Beaucoup de familles sont dans l'aveuglement et le mutisme. Il leur faut parfois des années pour identifier les conduites liées aux drogues du jeune. Quand elles ne peuvent plus ne pas les voir, elles vivent un sentiment de culpabilité qui les paralyse et elles s'isolent. Prises dans la honte, elles dissimulent la situation et elles supportent les transgressions du jeune jusqu'au degré ultime de l'insupportable. Elles peuvent être aussi trop déprimées pour pouvoir sortir de chez elles, « $y$ croire » et adresser un appel à l'extérieur.

Quand un certain degré de transgression est atteint, on voit des familles interpeller la justice, ce qui ne semble pas toujours les aider. Lorsque les comportements sans limites des jeunes, et leurs usages de drogues en particulier, sont sanctionnés par la loi, les parents observent comment les risques judiciaires se conjuguent aux risques sanitaires et sociaux et provoquent une intensification des conduites d'(auto)destruction et des transgressions de leurs enfants. Le traumatisme carcéral en particulier a un impact sur les trajectoires psychologiques, sanitaires, relationnelles et sociales qui peut être irréversible. La justice peut-elle prendre en charge les troubles de socialisation des jeunes et la complexité des demandes que lui adressent les familles? N'offrir comme réponses aux itinéraires de risques de la jeunesse que la répression et l'incarcération n'a aucun sens.

21 Tous les jeunes vivant dans mes sites d'enquête ne développent pas des comportements « décalés » et (auto)destructeurs. Ces comportements s'inscrivent dans le croisement de blessures intimes, de l'histoire évolutive des familles et des individus et d'un processus de socialisation particulier.

Traumatismes et intrigues familiales

De nombreuses familles, en interrogation devant les souffrances familiales, empruntent les chemins de la mémoire et du souvenir. Elles explorent leur histoire familiale. Les somatisations, les toxicomanies ou autres conduites à risques viennent parfois «montrer» des blessures insupportables, ce qui n'a pas pu être dit et entendu, les affects qui, parce qu'ils n'ont pas pu être identifiés, représentés et socialisés, réinscrivent constamment le drame dans la vie des individus et de leur filiation. Des familles racontent comment les mêmes séquences de mise en danger, de désespoir et de violence se rejouent en boucle depuis plusieurs générations dans la saga familiale. Le temps de ces familles semble arrêté sur le même synopsis, sans qu'elles en aient toujours conscience. Ces intrigues familiales sont souvent invisibles pour les protagonistes.

Chaque récit met en scène des trames narratives particulières. Les transgressions graves et les itinéraires de risques peuvent être des " écrans de fumée » qui masquent, certains enchaînements transgénérationnels: des carences affectives précoces, des ruptures familiales violentes à l'adolescence souvent liées à des difficultés 
d'émancipation, la peur et l'insécurité intérieure à l'âge adulte, l'angoisse sexuelle, la violence conjugale et les troubles des frontières entre les générations.

Les points d'appui des familles

24 Les récits montrent l'évolution des dynamiques familiales à travers le temps. Les situations ne sont pas toujours enkystées dans la répétition, elles évoluent en fonction des expériences de vie des personnes et des ressources qu'elles trouvent dans leur environnement. Il faut souvent de l'aide extérieure pour entrer dans des processus de changement. S'ils sont isolés les gens n'y arrivent pas, c'est dans la relation avec d'autres qu'ils se construisent autres. Mes interlocuteurs sont des êtres sociaux; s'ils ont pu "reprendre leur vie en main", c'est souvent grâce à la communauté qui les a progressivement étayés. Sous la pression des prises de risques démesurées et de la violence qui traversaient leur foyer, des familles mobilisent des ressources et des points d'appui auprès des professionnels, dans le groupe familial et l'environnement.

Beaucoup de familles ont pu "remettre de l'ordre» dans leurs relations familiales, instaurer des repères et un cadre normatif à leur vie quotidienne, en faisant appel aux représentants institutionnels (le monde scolaire, l'aide, les soins, la justice...) ou parce que des professionnels se sont rapprochées d'elles (le travail de proximité, les visites à domicile à des services sociaux des écoles, parfois même des formes triangulées d'aide contrainte ont favorisé ce rapprochement). Beaucoup trouvent certaines «solutions » dans le réseau social de proximité. De nombreuses personnes sont intervenues dans les trajectoires des familles rencontrées et se sont montrées adéquates (pharmacien, médecin généraliste, enseignante, assistante sociale de l'école, patron d'entreprise, responsables communal...). Leur savoir-faire relationnel a eu une action déterminante. Ces gens ont opéré un déplacement vers les familles, leur montrant une attention, une compréhension de leur condition et de leurs contextes de vie. Ils ont été des points d'écoute, de prévention et d'aide. Ils ont souvent été la première marche de l'accès aux ressources des dispositifs de soins. Certains ont même occupé, sur le long terme, des places structurantes dans la vie des jeunes et des familles pris dans des itinéraires de risques (rôle de tiers...).

Beaucoup de familles sont entrées dans un processus de changement "en se mélangeant ", parce qu'elles ont fait partie de cercles locaux. Des groupes de formation, d'auto-support, d'épargne collective, ethniques ou spirituels ont souvent joué un rôle de tiers dans les relations familiales. S'insérer dans des échanges sociaux (donner, recevoir, rendre ${ }^{14}$ ) a permis à de nombreuses interlocutrices de trouver des lieux d'écoute et d'expression, des protections et des substitutions qui ont fait évoluer leurs scénarios de vie. En participant à des groupes, elles sont sorties de l'isolement et ont développé leurs centres d'intérêt et leurs compétences symboliques, intellectuelles, techniques et sociales. Elles ont alors pu «desserrer» leurs liens familiaux et se construire une insertion sociale différente.

Pour vivre en paix sur mes sites d'enquête, il faut avoir un large réseau d'interconnaissance, «faire partie d'un cercle» ou au moins "se montrer avec des gens qui sont connus». D'autre part, l'argent est rare et difficile à épargner. Ces deux préoccupations quotidiennes expliquent le succès des cercles d'épargne collective : les «cagnottes» dans le milieu de «belges et mélangés » et les «guns» des femmes turques. Les cagnottes sont une forme d'épargne, hebdomadaire et "obligatoire ", qui réunit toutes les fins de semaine les participants au café, où la tirelire commune, la cagnotte, est installée. Chacun reste propriétaire de son capital, mais la somme globale déposée 
sur un compte commun produit des intérêts. Ils permettent d'organiser des repas de fête entre cagnotteurs. Leur épargne leur est remise pour les fêtes de fin d'année. Les cagnottes s'inscrivent dans la tradition ouvrière même si actuellement les cagnotteurs sont, pour la plupart, des personnes exclues du marché du travail licite. Cagnotter est une pratique économique et sociale qui permet d'entrer dans « une famille élargie » et d'étendre son réseau d'entraide et de solidarité.

Les "guns» sont une forme d'épargne collective propre aux femmes issues de l'immigration turque. Elles fonctionnent sur la parole donnée. Un réseau d'une dizaine d'amies se réunit tous les mois chez l'une d'entre elles. Elles mangent, dansent et discutent des relations conjugales, de la scolarité, de l'éducation des enfants... À cette occasion, chacune donne à l'hôtesse une somme d'argent convenue (en général 120 euros). « Faire gun » permet d'épargner mais, surtout, consolide les liens et permet de sortir des huis clos familiaux. Plus ces femmes font de "guns", meilleure est leur position dans la communauté.

29 Lorsque des comportements décalés et autodestructeurs traversent les foyers, l'expérience religieuse et l'appartenance à une communauté spirituelle peuvent être des repères. De nombreuses familles rencontrées ont cherché une aide dans les grandes religions traditionnelles : catholicisme, protestantisme ou islam. En renforçant leurs liens avec un tiers spirituel, elles prenaient distance par rapport à leur situation. Dans ces foyers sans pères, prêtres, pasteurs et imams ont parfois pris une fonction paternelle et suppléé à certains manques ${ }^{15}$. Des interlocuteurs se sont sentis écoutés par la divinité et ses représentants. Ils ont parfois reçu une aide concrète dans les situations de crise. Certains ont été hébergés dans les communautés religieuses, trouvant là un support social qui les a sortis de l'isolement.

L'enquête montre aussi que les groupes spirituels fondamentalistes peuvent être un leurre voire un espace de manipulation totalitaire. Dans un premier temps, ils sont un recours pour des jeunes et des familles, à bout de ressources, pris dans la détresse et les styles de vie à risques, mais les récits révèlent certaines dérives. Des responsables abusent de la place d'autorité qu'ils occupent dans la vie des personnes blessées, en quête de sens. En rendre compte est un enjeu démocratique. S'il y a peut-être là un droit d'ingérence de l'État, il y a aussi des responsabilités qu'il doit prendre. Les communautés de vie fondamentalistes offrent une écoute d'urgence et un accueil sans condition aux jeunes et aux familles en crise. Elles leur proposent des activités, des responsabilités et le support d'un groupe. Nos dispositifs peuvent-ils offrir des alternatives crédibles ? Il y a peut-être là des réponses à inventer. Pour une fraction de population, les services ne sont pas adaptés, ils ne répondent pas à leurs besoins immédiats et leur offrent des formes de soutien inapproprié ${ }^{16}$.

Conclusion

31 L'enclavement des populations les plus précarisées dans des zones ghettos, abandonnées par la puissance publique et l'économie mondialisée, montre une diminution de la capacité intégratrice de notre structure sociale. Les situations extrêmes des familles rencontrées donnent un relief, une lisibilité particulière, aux transformations familiales et sociales à l'œuvre dans l'ensemble de notre société. La marge et les conduites en apparence aberrantes éclairent les caractéristiques et les évolutions en cours dans notre société ${ }^{17}$. En bas de l'asymétrie sociale, l'ampleur des conduites d'(auto)destruction des jeunes et leurs activités liées aux drogues en particulier sont liées aux contextes de vie. Elles sont un mode d'adaptation au 
fonctionnement de leurs lieux de socialisation (famille, quartier, école). Les tensions incarnées dans la population pré-adulte sont un signal d'alarme. Cette enquête montre à quel point il devient difficile pour notre structure sociale d'inscrire l'ensemble de la jeunesse dans un système de références et d'échanges capable de l'inclure et de construire la paix sociale. Les récits posent, lancinante, la question de la construction des interdits sociaux et du devenir des normes dans un milieu populaire pris dans la tourmente post industrielle. Les «business» clandestins sont une appropriation locale particulièrement violente de notre modèle culturel ${ }^{18}$. Les jeunes "businessmen " des cités se bricolent des rêves de destins "hors normes ", "décalés » sur le plan de la légalité, mais en concordance avec les valeurs du néo-individualisme contemporain qui nous contraint à devenir les entrepreneurs de nos propres vies, à nous inventer nousmêmes dans le présent, dans un jeu social héroïque où la rhétorique est celle du combat pour s'arracher à sa condition. Les enjeux et règles du jeu de l'économie clandestine sont un des miroirs de la mondialisation ${ }^{19}$. La socialisation dans les réseaux du business n'est pas nécessairement l'humanisation. Les interdits de toute puissance et d'instrumentalisation de l'autre n'y sont pas nécessairement posés, pas plus qu'ils ne semblent l'être dans les logiques économiques du marché où la concurrence, l'affrontement et les critères de profits sont des valeurs supérieures au respect de l'humain. Les codes sociaux de l'économie de marché peuvent-ils humaniser la jeunesse? La question est béante partout où la mondialisation a implanté son modèle culturel.

Pourtant, malgré le poids du passé et l'état de délabrement de la structure sociale, sous la pression des conduites à risques qui les traversent, des familles sortent du mutisme et de l'isolement. En se rapprochant des dispositifs d'aide et grâce aux ressources de leur réseau social, elles bénéficient de régulations, de points d'appui qui peuvent infléchir leurs trajectoires de risques et de précarisation. Beaucoup ont fait évoluer leurs conditions de vie et sont entrées dans un processus de changement. Certaines ont fait des apprentissages relationnels et sociaux dans les groupes spirituels, d'épargne, sportifs, d'entraide, de formation ou professionnels. Ils ont été des cercles d'entraide et de solidarité de première importance. Pour faire face aux difficultés qui la traversent, la communauté est inventive, elle s'organise, crée ou utilise des supports adaptés aux traditions et aux évolutions des conditions de vie. Des interlocuteurs font remarquer que les professionnels de l'intervention sanitaire, sociale et éducative gagneraient à «se mélanger » aux pratiques sociales spécifiques existant dans les quartiers. Participer aux cercles d'épargne collective, aller à la rencontre des groupes spirituels qui sont un support pour de nombreuses familles, engager un travail de proximité en leur direction (à partir du milieu scolaire, du travail de quartier, des «visites à tables " en milieu carcéral...) permettraient de réduire « les écarts » de mentalité. Les politiques d'aide et de prévention seront d'autant plus adaptées qu'elles créeront des espaces de rencontre avec les familles qui se tiennent à distance des institutions, tisseront des liens, des alliances et des partenariats sur les lieux de vie. Pour rapprocher les services des familles en difficultés, il est nécessaire de décloisonner les "bulles sociales" dans lesquels les uns et les autres vivent.

33 J'ai cherché à rendre compte de ce que les familles qui ont participé à l'enquête m'ont appris. Les récits de vie s'inscrivent dans « la force de la chose donnée : cette vertu qui pousse les dons à circuler, à être donnés et à être rendus " ${ }^{20}$. Ce qu'on reçoit, on ne le garde pas pour soi, on le transmet. Cet article est un des supports de la circulation de la chose donnée. Il est construit à partir des récits des personnes qui ont participé à 
l'enquête. Ils se sont répondus et mêlés jusqu'à construire un savoir empirique, contextualisé et transversal sur les parcours de risques des jeunes ${ }^{21}$.

\section{NOTES}

1.. P. JAMOULLE, La Débrouille des familles. Récits de vies traversés par les drogues et les conduites à risques, De Boeck Université, coll. « Oxalis », 2002.

2.. «L'objectif principal de l'ethnographe est de rendre intelligible à un public aussi large que possible l'expérience d'êtres humains telle que leur appartenance à un groupe social contribue à les déterminer », D. SERBER, cité par J.-F. WERNER dans Marge, sexe et drogues à Dakar. Enquête ethnographique, Paris, Karthala-Orstom, 1993.

3.. Voir à ce sujet les réflexions méthodologiques de Lise Causse et Pierre Roche dans le rapport de recherche sur la prévention de proximité de la Mission Sida Toxicomanie Ville de Marseille/Céreq, Janvier 2002.

4.. Voir l'article de Michel JOUBERT : « Drogues, trafics et insertion, l'économie informelle comme support social ", Cahier de prospective jeunesse, Vol. $5, \mathrm{n}^{\circ} 3$, trimestre 00.

5.. Dévoilé dans Le Culte de la performance, d'Alain EHRENBERG, Calmann-Lévy, 1991.

6.. Je partage les thèses des interactionnistes qui analysent les processus de socialisation comme des modes d'adaptation et d'ajustements progressifs aux contextes de vie. Mes propres enquêtes n'ont cessé de m'enseigner que face aux situations rencontrées, l'individu tente d'ajuster son comportement au mieux de ses préférences et de ses intérêts tels qu'il les conçoit. Les processus de socialisation ne relèvent pas du simple conditionnement mais bien d'une adaptation progressive au champ d'interaction dans lequel l'acteur est plongé.

En ce sens, cette citation de Mike SINGLETON est explicite : « Les structures, les fonctions, les systèmes, les modèles, les valeurs et les visions culturelles, les faits sociaux (totaux ou partiels) ne sont pas des facteurs qui pèsent du dehors et d'en haut, de tout leur poids, indépendant et pré-existant, sur les acteurs individuels. Ils ne sont ni l'eau dans laquelle le poisson baigne, sans le savoir ni l'eau qui coule sur le dos d'un canard imperméable. En fait, il n'y a que des personnes qui en se socialisant se retrouvent socialisées. » (Amateur de chiens à Dakar, plaidoyer pour un interprétariat anthropologique, Louvain-la-Neuve, Paris, Academia-Bruylandt/L'Harmattan, 1998).

7.. Voir à ce sujet l'ouvrage de David LEPOUTRE, Cour de banlieue. Codes, rites et langages, Paris, Odile Jacob, 2001, ainsi que le chapitre sur les systèmes de normes qui structurent les sociabilités souterraines, développé dans un de mes précédents ouvrages : Drogues de rue, récits et styles de vie, Bruxelles-Paris, De Boeck, coll. « Oxalis », 2000.

8.. Joutes verbales, sous forme de plaisanteries, et d'histoires moqueuses à rebondissements.

9.. Violence gratuite.

10.. Les travaux de Vincent de Gaulejac montrent que dans la société dans son ensemble, la lutte des places se substitue à la lutte des classes. 
Vincent de GAULEJAC, « De la lutte des classes à la lutte des places », dans Précarisation du travail et lien social. Des hommes en trop ?, sous la dir. de Frédéric ABÉCASSIS et Pierre ROCHE, L'Harmattan, 2001.

11.. Tout au long de l'étude de terrain, le regard posé sur les conduites à risques resta essentiellement féminin. Malgré mes efforts pour diversifier mes modes d'approche et entrer en contact avec des pères et des frères concernés, mes interlocuteurs sont restés principalement des femmes (grands-mères, mères ou sœurs). Trente-trois personnes se sont engagées dans des récits de vie approfondis. Parmi elles, il n'y avait que sept hommes. N'avoir presque exclusivement que des femmes comme interlocutrices est une situation d'enquête très particulière que je n'avais jamais rencontré précédemment. Elles semblaient être les seules à pouvoir nommer ce qui se passait dans les familles.

12.. Voir à ce sujet les travaux de Paul ALÉRINI, Précarisation du travail et lien social des hommes en trop ?, op. cit, 2001.

13.. En milieu populaire tout particulièrement.

14.. M. MAUSS, « Essai sur le don », Sociologie et anthropologie, Quadrige/PUF, 1950.

15.. Voir à ce sujet l'ouvrage Structuration psychique de l'expérience religieuse, la fonction paternelle de Vassilis SAROGLOU, Paris, L'Harmattan, 1997.

16.. Voir à ce sujet les travaux de l'association française RESSCOM ainsi que l'ouvrage Drogues de rue. Récits et styles de vie, P. JAMOULLE, 2000.

17.. « Dans toutes ces conduites en apparence aberrantes, les "malades" ne font que transcrire un état du groupe et rendre manifeste telle ou telle de ses constantes. Leur position périphérique par rapport à un système local n'empêche pas qu'au même titre que lui, ils ne soient partie intégrante du système total », C. LÉVI-STRAUSS, « Introduction à l'œuvre de Marcel Mauss », in M. Mauss, sociologie et anthropologie, Quadrige/PUF, 1950. 18..Voir à ce sujet les ouvrages d'Alain EHRENBERG, Le Culte de la performance, Paris, Calmann-Lévy, 1991 et La Fatigue d'être soi, Paris, Odile Jacob, 1999.

19.. Dans leur magnifique article, « Réflexion sur la jeunesse », Jean et John COMAROFF montrent que les conduites « décalées » des jeunes dans les situations d'asymétrie sociale sont inhérentes à la diffusion du modèle néo-libéral d'origine nord-américaine. À Los Angeles, Dakar, Londres ou Delhi, elles prendraient une forme homogène ou identique. «Beaucoup de jeunes entrepreneurs, élevés dans la culture de marché et de la marchandise trouvent leurs propres voies et moyens. [...] En nombre sans cesse grandissant, ils entrent dans les réseaux du trafic international de stupéfiants et/ou sombrent dans un monde de ténèbres où l'usage de violence devient un mode routinier de production et de redistribution - des pratiques qui souvent rappellent le business international et qui érodent visiblement l'autorité de l'État. [...] Faut-il s'étonner dès lors si notre adolescent - avec ses chaussures de sport à prix exorbitant, son baladeur lui crachant aux oreilles un rap décoiffant et son bipeur qui le relie aux réseaux souterrains de l'économie mondialisée - est une synthèse si réussie de l'enfant des rues et du nabab de l'économie ? » « Réflexions sur la jeunesse, du passé à la postcolonie », Revue Politique Africaine $\mathrm{n}^{\circ}$ 80, déc. 2000.

20.. M. MAUSs, op. cit.

21.. « Au fond ce sont des mélanges. On mêle les âmes dans les choses; on mêle les choses dans les âmes. On mêle les vies et voilà comment les personnes et les choses mêlées sortent chacune de sa sphère et se mêlent : ce qui est précisément le contrat et l'échange. » M. MAUss, op. cit. 
Bibliographie : F. ABECASSIS et P. ROCHE (dir.), Précarisation du travail et lien social. Des hommes en trop ?, Paris, L'Harmattan, 2001 ; S. AQUATIAS et E. JACOB, Usages de psychotropes et prévention des conduites à risques, Conseil Général de Seine Saint-Denis, 1998 ; P. BOUHNIK, Le Monde social des usagers de drogues dures en milieu urbain défavorisé, Thèse de doctorat en sociologie, Université de Paris VIII, 1995 ; Ph. BOURGOIS, En quête de respect, le crack à New York, Paris, Seuil, 2001 [1995] ; Jean et John COMAROFF, Réflexions sur la jeunesse, du passé à la postcolonie, revue Politique Africaine $n^{\circ} 80$, décembre 2000 ; A. EHRENBERG, Le Culte de la performance, Paris, Calmann-Lévy, 1991 ; A. EHRENBERG, La Fatigue d'être soi, Paris, Odile Jacob, 1999 ; E. GOFFMAN, Stigmate. Les usages sociaux des handicaps, Paris, Éditions de Minuit, 1975 [1963] ; J.-P. JACQUES, Pour en finir avec les toxicomanies. Psychanalyse et pourvoyance légalisée des drogues, Paris-Bruxelles, De Boeck Université, 1999 ; P. JAMOULLE, La Débrouille des familles. Récits de vie traversés par les drogues et les conduites à risques, Bruxelles-Paris, De Boeck, coll. « Oxalis », 2002, P. JAMOULLE, Drogues de rue, récits et styles de vie, Bruxelles-Paris, De Boeck, coll. « Oxalis », 2000 ; P. JAMOULLE, Parents usagers de drogues, rapport de recherche de l'Atelier Parentalité et usages de drogues en Communauté française, Communauté française de Belgique, 1998 ; P. JAMOULLE, Prévention des conduites à risques : la question des toxicomanies, Prévenir, $\mathrm{n}^{\circ} 31$; P. JAMOULLE, Circulation des drogues et réseaux de sociabilité de l'économie souterraine, l'Observatoire, n²3, 1999 ; P. JAMOULLE, N. PANUNZI-ROGER, Les Conduites liées aux drogues dans les zones de précarité, Enquête de terrain auprès de professionnels, Psychotropes, vol. 7, n 3-4, 2001 ; P. JAMOULLE, « Les conduites liées aux drogues dans les zones de précarité, Enquête de terrain auprès d'usagers de drogues ", Psychotropes, vol. 7, n 3-4, 2001 ; P. JAMOULLE, «Styles de vie liés aux drogues et trajectoires de sortie de toxicomanie, Enquête sur le site belge (Hainaut) », Psychotropes, vol. 7, n 3-4, 2001 ; M. JOUBERT (dir.), Précarisation risque et santé, INSERM, Questions de santé publique, 2001 ; M. JOUBERT, Drogues, trafics et insertion, l'économie informelle comme support social, Cahier de Prospective jeunesse, Vol. $5 \mathrm{n}^{\circ} 3$, trimestre 2000 ; J.-F. LAE, N. MURARD, L'Argent des pauvres. La vie quotidienne en cité de transit, Paris, Le Seuil, 1985 ; J.-F. LAE, N. MURARD, Les Récits du malheur, Paris, Descartes \& Cie, 1995 ; J.-F. LAE, A. FARGE, Fracture sociale, Paris, Desclée de Brouwer, 2000 ; P. LEGENDRE, Leçon IV. Les enfants du texte. Étude sur la fonction parentale des états, Paris, Fayard, 1992 ; D. LEPOUTRE, Cour de banlieue, Codes, rites et langages, Paris, Poche Odile Jacob, 2001 (1997) ; M. MAUSs, « Essai sur le don », in Sociologie et anthropologie, Quadrige/PUF, 1950 ; M. SINGLETON, Amateur de chiens à Dakar, plaidoyer pour un interprétariat anthropologique, Louvain-la-Neuve, Paris, AcademiaBruylandt/l'Harmattan, 1998 ; J.-F. WERNER, Marges, sexe et drogues à Dakar, enquête ethnographique, Paris, Karthala-Orstom, 1993.

\section{RÉSUMÉS}

Cet article est une étude ethnographique qui résulte des récits de vie récoltés auprès de familles belges de milieu populaire, traversées par des conduites à risques (violences, micro-trafics, tentatives de suicide, addictions...). Il apparaît que des familles sortent du mutisme et de 
l'isolement et que beaucoup ont fait évoluer leurs conditions de vie et sont entrées dans un processus de changement.

This ethnographical study is the result of series of investigations and dialogs with Belgian families. They have in common their appurtenance to a popular surroundings and a difficult situation: violence, illegal traffics, suicidal attempts, drug addictions... It seems that these families try to evolve and change their life conditions to a better way. 